International Journal of Pure and Applied Mathematics

Volume 96 No. 3 2014, 299-306

ISSN: 1311-8080 (printed version); ISSN: 1314-3395 (on-line version)

url: http://www.ijpam.eu

doi: http://dx.doi.org/10.12732/ijpam.v96i3.1

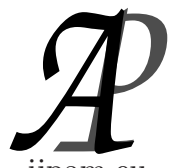

ijpam.eu

\title{
ON THE MAZUR-ULAM THEOREM IN FUZZY ANTI-2-NORMED SPACES
}

\author{
Majid Abrishami-Moghaddam \\ Department of Mathematics \\ Birjand Branch \\ Islamic Azad University \\ Birjand, IRAN
}

\begin{abstract}
In this article, we study the notions of 2-isometries in fuzzy anti-2normed spaces and prove a Mazur-Ulam type theorem in the 2-strictly convex fuzzy anti-2-normed spaces.
\end{abstract}

AMS Subject Classification: 46S40, 39B52, 39B82, 26E50, 46S50

Key Words: fuzzy anti-2-normed space, Mazur-Ulam theorem, 2-strictly convex, 2-collinear

\section{Introduction}

The theory of fuzzy sets was introduced by L. Zadeh [14] in 1965 and thereafter several authors applied it different branches of pure and applied mathematics. Many mathematicians considered the fuzzy normed spaces in several angels (see [3], [10], [13]). In [8] Iqbal H. Jebril and Samanta introduced fuzzy anti-norm on a linear space depending on the idea of fuzzy anti-norm was introduced by Bag and Samanta [1] and investigated their important properties.

Let $X$ and $Y$ be metric spaces with metrics $d_{X}$ and $d_{Y}$, respectively. A map $f: X \rightarrow Y$ is called an isometry if $d_{Y}(f(x), f(y))=d_{X}(x, y)$ for every $x, y \in X$. In 1932 , the theory of isometric mappings was originated in the classical paper

Received: January 25, 2014

(c) 2014 Academic Publications, Ltd. url: www.acadpubl.eu 
[11] by Mazur and Ulam. They have proved the following theorem.

Mazur-Ulam Theorem. Let $f$ be an isometric transformation from a real normed vector space $X$ onto a real normed vector space $Y$ with $f(0)=0$. Then $f$ is linear.

The theorem is not true for normed complex vector spaces. It was a natural ask if the theorem holds without the onto assumption. In fact, the hypothesis of surjectivity is essential. Without this assumption, Baker [2] proved that every isometry from a normed real space into a strictly convex normed real space is linear up to translation. Chu et al. [4] have defined the notion of a 2-isometry which is suitable to represent the concept of an area preserving mapping in linear 2normed spaces. In [5], Chu proved that the Mazur-Ulam theorem holds in linear 2-normed spaces under the condition that a 2-isometry preserves collinearity. Chu et al. [6] discussed characteristics of 2-isometries. Recently, some authors have studied the Mazur-Ulam theorem on non-Archimedean normed linear spaces [9], [12]. In this paper, we prove that the Mazur-Ulam theorem holds under some conditions in the fuzzy anti-2-normed spaces. We establish a Mazur-Ulam type theorem in the framework of strictly convex normed spaces by using some ideas of [7]. Now we recall some notations and definitions used in this paper.

\section{Preliminaries}

Definition 2.1. Let $X$ be a linear space over a real field $F$. A fuzzy subset $N$ of $X \times X \times \mathbb{R}$ is called a fuzzy anti-2-norm on $X$ if the following conditions are satisfied for all $x, y \in X$ :

$\left(a-N_{1}\right)$ For all $t \in \mathbb{R}$ with $t \leq 0, N(x, y ; t)=1$,

$\left(a-N_{2}\right)$ For all $t \in \mathbb{R}$ with $t>0, N(x, y ; t)=0$ if and only if $x$ and $y$ are linearly dependent,

$\left(a-N_{3}\right)$ For all $t \in \mathbb{R}$ with $t>0$ and $x, y \in X, N(x, y ; t)=N(y, x ; t)$,

$\left(a-N_{4}\right)$ For all $t \in \mathbb{R}$ with $t>0, N(\alpha x, y ; t)=N(x, y ; t /|\alpha|)$, for all $\alpha \neq 0, \alpha \in F$,

$\left(a-N_{5}\right)$ For all $s, t \in \mathbb{R}, N(x+y, z ; t+s) \leq \max \{N(x, z, t), N(y, z, s)\}$,

$\left(a-N_{6}\right) N(x, y, t)$ is a non-increasing function of $t \in \mathbb{R}$ and $\lim _{t \rightarrow \infty} N(x, y, t)$ $=0$.

Then the pair $(X, N)$ is called a fuzzy anti-2-normed linear space. 
Example 2.2. Let $(X,\|.,\|$.$) be a 2-normed space. If we define$

$$
N(x, y ; t)=\left\{\begin{array}{ccc}
\frac{\|x, y\|}{t+\|x, y\|} & \text { if } \quad t>0 \\
1 & \text { if } \quad t \leq 0 .
\end{array}\right.
$$

then $(X, N)$ is called the standard fuzzy anti-2-norm induced by the 2-norm $\|.,$.$\| .$

Definition 2.3. A fuzzy anti-2-normed space $X$ is called 2-strictly convex if $N(x+y, z, s+t)=\max \{N(x, z, s), N(y, z, t)\}$ and $N(x, z, s)=N(y, z, t)$ implies that $x=y$ and $s=t$.

Definition 2.4. Let $(X, N)$ and $(Y, N)$ be two fuzzy anti-2-normed spaces. We call that $f:(X, N) \rightarrow(Y, N)$ is a fuzzy 2-isometry if $N(x-z, y-z, t)=$ $N(f(x)-f(z), f(y)-f(z), t)$ for all $x, y, z \in X$ and $t>0$.

Definition 2.5. Let $X$ be a real linear space and $x, y, z$ mutually disjoint elements of $X$. Then $x, y$ and $z$ are said to be 2-collinear if $y-z=r(x-z)$ for some real number $r$.

\section{Main Results}

In this section we will prove that the Mazur-Ulam theorem under some conditions in the fuzzy real anti-2-normed 2-strictly convex spaces. First, we prove some lemmas that is require for the main theorem of our paper.

Lemma 3.1. Let $(X, N)$ be a fuzzy anti-2-normed space. Then

$$
N(x, y, t)=N(x, y+r x, t) \quad \text { for all } r \in \mathbb{R} .
$$

Proof. Let $x, y \in X$ and let $r \in \mathbb{R}$. Without loss of generality, we may assume $s, t>0$. Then

$$
N(x, y+r x, t) \leq \max \{N(x, y, t), N(x, r x, t)\}=N(x, y, t) .
$$

Conversely,

$$
N(x, y, t)=N(x, y+r x-r x, t)
$$




$$
\begin{aligned}
& \leq N(x, y+r x-r x, t+s) \\
& \leq \max \{N(x, y+r x, t), N(x,-r x, s)\} \\
& =N(x, y+t x, t) .
\end{aligned}
$$

Thus $N(x, y, t)=N(x, y+r x, t)$ for all $r \in \mathbb{R}$.

Lemma 3.2. Let $(X, N)$ be a fuzzy anti-2-normed space which is strictly convex and let $x, y, z \in X$ and $t>0$. Then $u=\frac{x+y}{2}$ is unique element of $X$ such that

$$
N(x-z, x-u, t)=N(x-z, y-z, 2 t)
$$

and

$$
N(y-u, y-z, t)=N(x-z, y-z, 2 t),
$$

and $x, y, u$ are 2-collinear.

Proof. First, we prove that $x, y, u$ are 2-collinear. Since $u=\frac{x+y}{2}$ we have

$$
\begin{aligned}
x-u & =x-\frac{x+y}{2}=\frac{x}{2}-\frac{y}{2}=\frac{x+y-y}{2}-\frac{y}{2} \\
& =-\left(y-\frac{x+y}{2}\right)=-(y-u)
\end{aligned}
$$

Thus $x, y$ and $u$ are 2colinear. By using lemma (3.1), we have

$$
\begin{aligned}
N(x-z, x-u, t) & =N\left(x-z, x-\frac{x+y}{2}, t\right) \\
& =N(x-z, x-y, 2 t) \\
& =N(x-z, y-z, 2 t),
\end{aligned}
$$

and similarly $N(y-u, y-z, t)=N(x-z, y-z, 2 t)$. To show the uniqueness, assume that $v \in X$, satisfies the above properties. Since $x, y$ and $v$ are 2colinear, there exists a real number $s$ such that $v:=s x+(1-s) y$. In view of lemma (3.1) and definition (2.3), we obtain

$$
\begin{aligned}
N(x-z, y-z, 2 t) & =N(x-z, x-v, t) \\
& =N(x-z, x-(s x+(1-s) y), t) \\
& =N\left(x-z, x-y, \frac{t}{|1-s|}\right) \\
& =N\left(x-z, y-z, \frac{t}{|1-s|}\right) .
\end{aligned}
$$


Thus $2 t=\frac{t}{|1-s|}$. Since $t>0,|1-s|=\frac{1}{2}$. Also,

$$
\begin{aligned}
N(x-z, y-z, 2 t) & =N(y-v, y-z, t) \\
& =N(y-(s x+(1-s) y), y-z, t) \\
& =N\left(x-y, y-z, \frac{t}{|s|}\right) \\
& =N\left(x-z, y-z, \frac{t}{|s|}\right) .
\end{aligned}
$$

So, $2 t=\frac{t}{s \mid}$. Hence $\frac{1}{2}=|s|=|1-s|$ and so $s=\frac{1}{2}$. Therefore we obtain that $u=v$ and this complete the proof.

Lemma 3.3. Let $f:(X, N) \rightarrow(Y, N)$ is a fuzzy 2-isometry;

(i) For every $x, y, z, u \in X$, if $x, y$ and $z$ are 2-colinear, then $f(x), f(y)$ and $f(z)$ are 2-colinear.

(ii) If $f(0)=0$, then for every $x, y \in X$ and $t>0$

$$
N(x, y, t)=N(f(x), f(y), t) .
$$

Proof. ( $i$ ) Since $x, y$ and $z$ are 2-colinear, there exists a real number $s$ such that $y-x=s(z-x)$. So we have

$$
\begin{aligned}
N(f(y)-f(x), f(u)-f(x), t) & =N(y-x, u-x, t) \\
& =N(s(z-x), u-x, t) \\
& =N\left(z-x, u-x, \frac{t}{|s|}\right) \\
& =N\left(f(z)-f(x), f(u)-f(x), \frac{t}{|s|}\right) \\
& =N(s(f(z)-f(x)), f(u)-f(x), t)
\end{aligned}
$$

and by definition (2.3), we have that $f(y)-f(x)=s(f(z)-f(x))$.

To prove (ii), we have

$$
\begin{aligned}
N(x, y, t) & =N(x-0, y-0, z-0, t) \\
& =N(f(x)-f(0), f(y)-f(0), t) \\
& =N(f(x), f(y), t) .
\end{aligned}
$$

Theorem 3.4. Every fuzzy 2-isometry $f:(X, N) \rightarrow(Y, N)$ is affine. 
Proof. Let $g(x):=f(x)-f(0)$. Then $g$ is fuzzy 2-isometry and $g(0)=0$. Hence, it is enough to show that $g$ is linear. Let $x, y \in X$. By $(i)$ of Lemma (3.3), we obtain that $g\left(\frac{x+y}{2}\right), g(x)$ and $g(y)$ are 2 colinear. Now, from Lemma (3.2), for all $x, y \in X$, we have

$$
g\left(\frac{x+y}{2}\right)=g\left(\frac{x}{2}\right)+g\left(\frac{y}{2}\right)
$$

It follows that $g$ is $\mathbb{Q}$-linear. We have to show that $g$ is $\mathbb{R}$-linear.

Let $r \in \mathbb{R}^{+}$with $r \neq 1$ and $x \in X$. By $(i)$ of Lemma (3.3), since $0, x$ and $r x$ are 2-collinear $g(0), g(x)$ and $g(r x)$ are also 2-collinear. Since $g(0)=0$, there exists $r^{\prime} \in \mathbb{R}$ such that $g(r x)=r^{\prime} g(x)$. Now, we will proved that $r=r^{\prime}$. From (ii) of Lemma (3.3), for each $y \in X$ and $t>0$ we can write

$$
\begin{aligned}
N\left(x, y, \frac{t}{r}\right) & =N(r x, y, t) \\
& =N(g(r x), g(y), t) \\
& =N\left(r^{\prime} g(x), g(y), t\right) \\
& =N\left(g(x), g(y), \frac{t}{\left|r^{\prime}\right|}\right) \\
& =N\left(x, y, \frac{t}{\left|r^{\prime}\right|}\right) .
\end{aligned}
$$

So, $r= \pm r^{\prime}$. We assume that $r^{\prime}=-r$, that is, $g(r x)=-r g(x)$. Then there exists $q_{1}, q_{2} \in \mathbb{Q}$ such that $0<q_{1}<r<q_{2}$. For each $y \in X$, we have

$$
\begin{aligned}
N\left(g(x), g(y)-g\left(q_{2} x\right), \frac{t}{q_{2}+r}\right) & =N\left(q_{2} g(x)-(-r g(x)), g(y)-g\left(q_{2} x\right), t\right) \\
& =N\left(g(r x)-g\left(q_{2} x\right), g(y)-g\left(q_{2} x\right), t\right) \\
& =N\left(r x-q_{2} x, y-q_{2} x, t\right) \\
& =N\left(x, y-q_{2} x, \frac{t}{q_{2}-r}\right) \\
& \geq N\left(x, y-q_{2} x, \frac{t}{q_{2}-q_{1}}\right) \\
& =N\left(q_{1} x-q_{2} x, y-q_{2} x, t\right) \\
& =N\left(g\left(q_{1} x\right)-g\left(q_{2} x\right), g\left(y-q_{2} x\right), t\right) \\
& =N\left(g(x), g\left(y-q_{2} x\right), \frac{t}{q_{2}-q_{1}}\right)
\end{aligned}
$$


By $\left(a-N_{6}\right)$, we have $q_{2}+r \leq q_{2}-q_{1}$ which is a contradiction. Hence $r=r^{\prime}$, that is, $g(r x)=r g(x)$ for all positive real numbers $r$. Hence for every real number $r, g(r x)=r g(x)$. Therefore $f=g-g(0)$ is $\mathbb{R}$-linear and the proof is complete.

\section{References}

[1] T. Bag, S. K. Samanta, A comparative study of fuzzy norms on a linear space, Fuzzy Sets. Syst, 159 (2008), 670-684.

[2] J.A. Baker, Isometries in normed spaces, Amer. Math. Monthly, 78 (1971), 655-658.

[3] S.C. Cheng, J.N. Mordeson, Fuzzy linear operator and fuzzy normed linear spaces, Bull. Calcutta Math. Soc. (1994), 429-436.

[4] H. Y. Chu, C. K. Park, W. K. Park, The Aleksandrov problem in linear 2-normed spaces, J. Math. Anal. Appl. 289 (2004), 666-672.

[5] H. Y. Chu, On the Mazur-Ulam problem in linear 2-normed spaces, $J$. Math. Anal. Appl. 327 (2007), 1041-1045.

[6] H. Y. Chu, S. H. Ku, D.S. Kang, Characterizations on 2-isometries, J. Math. Anal. Appl. 340 (2008), 621-628.

[7] M. Eshaghi Gordji, S. Abbaszadeh, Th. M. Rassias, On the MazurUlam theorem in fuzzy $n$-normed strictly convex spaces, arXiv:0909.5675v1 (2009).

[8] Iqbal H. Jebril, T.K. Samanta, Fuzzy Anti-Normed space, J. Math. Tech., February (2010), 66-77.

[9] D. Kangb, H. Kohb, I. G. Choa, On the Mazur-Ulam theorem in nonArchimedean fuzzy normed spaces, Appl. Math. Let. 25, (2012), 301-304.

[10] A. K. Katsaras, Fuzzy topological vector spaces, Fuzzy Sets Syst, 12 (1984), 143-154.

[11] S. Mazur, S. Ulam, Sur les transformation isomtriques despaces vectoriels norms, C. R. Acad. Sci. Paris, 194 (1932), 946-948.

[12] M. S. Moslehian, Gh. Sadeghi, A Mazur-Ulam theorem in nonArchimedean normed spaces, Nonlinear Anal. 69 (2008), 3405-3408. 
[13] R. Saadati, S.M. Vaezpour, Some results on fuzzy Banach spaces, J. Appl. Math. Computing, 17 (2005) 475-484.

[14] L.A. Zadeh, Fuzzy sets, Inf. \& Control. 8 (1965), 338-35. 